\title{
A framework for simulation of aircraft flyover noise through a non-standard atmosphere
}

\author{
Michael Arntzen ${ }^{1}$ \\ National Aerospace Laboratory (NLR), 1059CM Amsterdam, the Netherlands \\ Stephen A. Rizzi ${ }^{2}$ \\ NASA Langley Research Center, Hampton, VA 23681 \\ Hendrikus G. Visser ${ }^{3}$ and Dick G. Simons ${ }^{4}$ \\ TU Delft, 2600 AA Delft, the Netherlands
}

\begin{abstract}
This paper describes a new framework for the simulation of aircraft flyover noise through a non-standard atmosphere. Central to the framework is a ray-tracing algorithm which defines multiple curved propagation paths, if the atmosphere allows, between the moving source and listener. Because each path has a different emission angle, synthesis of the sound at the source must be performed independently for each path. The time delay, spreading loss and absorption (ground and atmosphere) are integrated along each path, and applied to each synthesized aircraft noise source to simulate a flyover. A final step assigns each resulting signal to its corresponding receiver angle for the simulation of a flyover in a virtual reality environment. Spectrograms of the results from a straight path and a curved path modeling assumption are shown. When the aircraft is at close range, the straight path results are valid. Differences appear especially when the source is relatively far away at shallow elevation angles. These differences, however, are not significant in common sound metrics. While the framework used in this work performs off-line processing, it is conducive to real-time implementation.
\end{abstract}

\section{Nomenclature}

$\begin{array}{ll}c_{\text {eff }} & =\text { effective speed of sound } \\ f & =\text { frequency } \\ L_{\text {diff }} & =\text { loss due to diffraction } \\ R & =\text { molar gas constant } \\ T & =\text { temperature } \\ \mathrm{V}_{\mathrm{w}} & =\text { wind velocity } \\ \gamma & =\text { adiabatic index } \\ \psi & =\text { propagation direction } \\ \varphi & =\text { wind direction }\end{array}$

\section{Introduction}

$\mathrm{D}$

ESPITE the fact that aircraft noise has been reduced over the years, new designs for even quieter aircraft are being considered. ${ }^{1,2}$ Because annoyance to both current and future aircraft may not be well captured by integrated noise metrics, there is a need for subjective assessments of individual flyovers. While many empirical and physics based methods are available to compute component and system noise, ${ }^{3-6}$ their output is generally geared at producing frequency domain predictions or noise contours which are not well suited for subjective evaluation. Therefore, prediction-based noise synthesis is required to evaluate new designs (inclusive of changes to both source

\footnotetext{
${ }^{1} \mathrm{PhD}$ Candidate, Environment and Policy Support, Anthony Fokkerweg 2

${ }^{2}$ Senior Research Engineer, Structural Acoustics Branch, MS 463, AIAA Associate Fellow

${ }^{3}$ Associate Professor, Air Transport \& Operations, Kluyverweg 1, Delft, AIAA Associate Fellow

${ }^{4}$ Full Professor, Air Transport \& Operations, Kluyverweg 1, Delft
} 
and operations) based on subjective measures. Subjective evaluations of aircraft flyover noise are not common in the design phase, but can give valuable information regarding the impact of design changes, e.g. alternative configurations or operations.

Simulation of aircraft flyover noise is also an effective tool to communicate noise impact to stakeholders. Community noise is a global topic as airport authorities are confronted with the conflicting requirements of more stringent noise regulations while increasing the number of operations. They need tools to communicate with neighbors of airports on aircraft noise. Neighboring communities often struggle to understand the impact of new aircraft, procedures or atmospheric conditions on their sound exposure, and would benefit from audible (and visual) demonstrations of future situations in addition to noise exposure maps. For example, simulation of new arrival or departure procedures ${ }^{7}$ would allow airports to explore ways of minimizing impact to the community whilst maximizing capacity.

A virtual reality environment for the simulation of aircraft flyover noise was first created by the National Aeronautics and Space Administration (NASA) using ground-based, monaural recordings of actual aircraft flyover events. ${ }^{8}$ To assess future designs and to pre-evaluate future operational changes or varying atmospheric conditions, aircraft source noise must be synthesized. To that end, subsequent developments were geared toward component noise synthesis of various source components (jet, fan, rotary wing) using frequency and time domain predictions, and to the flyover simulation thereof. ${ }^{9-12}$ Most recently, the National Aerospace Laboratory (NLR) described its latest efforts in creating an integrated tool chain ${ }^{13}$ inclusive of noise prediction and estimation of the effects of realistic atmospheric propagation.

The strength of the synthesis and simulation codes developed at both organizations is that measurements of flyover noise are not needed, unlike other recording-based methods. ${ }^{8,14}$ As such, the laboratory environment allows the controlled testing of a variety of parameters without the need for costly flight test measurements. The limitations to the approach are the necessity to have good source noise prediction tools for synthesis and the challenge to accurately capture the propagation effects in the simulation. In particular, curved propagation paths associated with any non-uniform atmosphere affect not only the integrated atmospheric and ground plane absorption, time delay, and spreading loss along the curved path, but also the emission angle and the receiver angle, which govern the source noise and where it appears to come from, respectively. To overcome these limitations, NASA and the NLR adopted a new framework to allow evaluation of novel source prediction tools, new aircraft designs, aircraft procedures and atmospheric propagation codes for aircraft operating in non-standard atmospheres. The new framework is common to both organizations to facilitate collaborative development.

This paper focuses on the creation of this new simulation framework. Details are provided with respect to the first working prototype and on the implemented propagation method. Future research directions are highlighted based on the conclusions from the current study.

\section{Simulation Framework}

\section{A. Review of the prior approach}

Until this time, there were two simulation frameworks in place at the NASA and NLR. The Community Noise Test Environment (CNoTE), ${ }^{9,10}$ was developed at NASA using a source-path-receiver paradigm. By treating the aircraft noise source as a compact acoustic source, the noise received at a listener in the far-field can be described as coming from a single emission angle. The emission angle, and its receiver angle complement, are determined by the instantaneous straight-line path between the source and listener. Source noise synthesis amounts to generating an emission angle specific pressure time history at the moving source, ${ }^{9-11}$ based on source directivities from predictive programs like ANOPP. ${ }^{15}$ The atmospheric propagation along the straight-line path takes into account time-varying spherical spreading loss, atmospheric and ground-plane absorption and time delay (simulating the Doppler effect). Finally, the receiver processing takes the listener orientation into account and applies binaural simulation using Head Related Transfer Functions (HRTF) ${ }^{16}$ for playback over headphones, or vector base amplitude panning $(\mathrm{VBAP})^{17}$ for playback over a loudspeaker array. The propagation and receiver processing stages are performed in real-time on the AuSIM GoldserverTM, a dedicated audio digital signal processing (DSP) computer. ${ }^{18}$ When coupled with the remaining elements of CNoTE, the listener is exposed to synthesized aircraft flyover noise within a 3-D virtual reality environment, inclusive of computer graphics visualization.

The NLR software, the Virtual Community Noise Simulator (VCNS), ${ }^{13}$ is a derivative of NASA CNoTE. The NLR uses the VCNS to demonstrate potential changes around airports to policy makers and to study aircraft noise impact in urban environments. The software is expanded in the sense that it contains a dedicated tool chain comprised of flight mechanics, source sound prediction and non-homogeneous atmospheric propagation. In a departure from the NASA approach, the sound is synthesized at the listener rather than at the source, in effect 
producing a pseudo-recording which is subsequently processed for 3-D presentation. Results from both the prior NASA and NLR frameworks are uniquely capable of presenting predicted flyover noise in an early design stage of an aircraft or new procedure.

\section{B. Requirements}

The goal of the new framework is, in the end, to allow real-time simulation of flyover noise created by an arbitrary aircraft flying an arbitrary trajectory, through an arbitrary atmosphere. While not presently performed, the prior frameworks would allow this with the exception of the arbitrary atmosphere. Including an arbitrary atmosphere adds the requirement to determine the curved path(s) as a function of time along the flight trajectory, and to compute and apply the integrated absorption, time delay and spreading loss along those paths.

The curved path influences the emission and receiver angles in a more complicated fashion than in the straightline propagation. As in the existing approach, the simulation framework should allow immersion of a test subject in a virtual reality environment to reproduce outdoor listening conditions as closely as possible. It should additionally allow the subject to affect the propagation path through his own movement. As will be discussed, the calculation of the curved path is computationally intensive and is presently not performed in real-time. Therefore an architecture was desired which allows off-line processing for present use, but is amenable to future real-time implementation with minor modifications.

\section{Atmospheric Propagation}

Most aircraft noise prediction models assume a homogeneous atmosphere where the sound waves, as a consequence, travel along straight paths. This disallows the formation of shadow zones and the potential for multiple, curved propagation paths from a source to a listener. These effects, however, do result from an inhomogeneous atmosphere where wind and/or temperature gradients cause refraction. The Fast Field Program (FFP $)^{19}$ method included in the NLR tool chain was initially explored to predict these effects, but proved to be too computationally expensive for its intended simulation use. ${ }^{13}$ Therefore a fast ray tracing method ${ }^{20}$ was developed that could deliver similar results at a fraction of the computational expense. Although there are more accurate models than ray tracing, ${ }^{21}$ they do not provide easy access to data necessary for noise synthesis, namely emission and receiver angles, and full traceable paths along which to integrate attenuation and time delay. Further, they are more computationally expensive.

\section{Ray tracing}

The ray tracing code is based on the numerical integration of Snell's law. ${ }^{20}$ The index of refraction is based on the effective speed of sound profile that simulates the atmospheric propagation effects. ${ }^{21}$ This assumption is generally valid if the atmospheric conditions do not change radically over one wavelength. A change in the index of refraction will lead to a change in direction of the ray path and, as a result, the entire path usually exhibits curvature. The effective sound speed, $c_{e f f}$, is the summation of the sound speed based on the temperature and the wind field in the direction of propagation, as in Eq. (1). It is further referred to in this paper as the sound speed profile.

$$
c_{e f f}=\sqrt{\gamma R T}+V_{w} \cos (\varphi-\psi)
$$

Here $\gamma$ is the adiabatic index, $R$ is the molar gas constant, $T$ is the (local) absolute temperature, $V_{w}$ is the local horizontal wind speed, and $\phi$ and $\psi$ specify the propagation and wind direction. In this way, the wind direction relative to the propagation direction is accounted for in the calculation of the sound speed profile. The azimuthal dependency of the wind field is thus collapsed in a sound speed profile that only changes with the altitude. This is sometimes referred to as a "2.5-dimensional" modeling approach.

In case of downwind propagation, i.e., for the wind blowing in the direction of sound propagation, the wind term is added to the temperature term. However, in case of upwind propagation, the wind term is subtracted from the temperature term. Consequently, the propagation effects in up- and downwind conditions differ as sketched in Figure 1. 


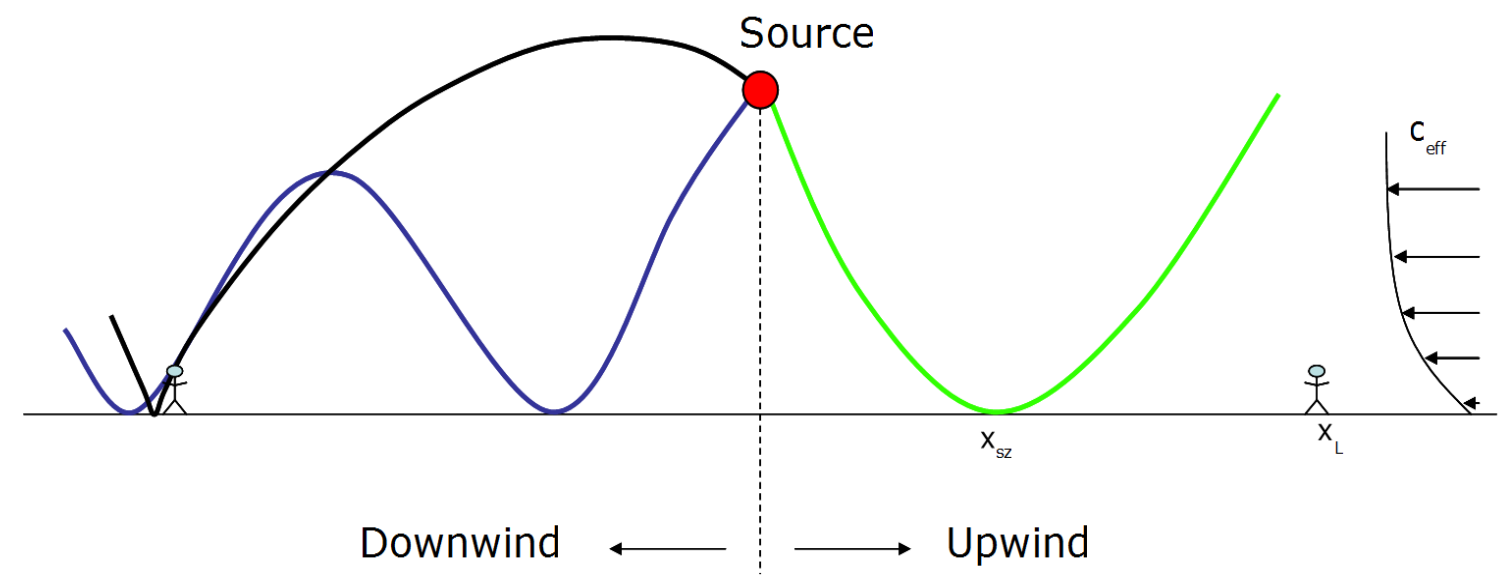

Figure 1 The qualitative effects of wind on ray paths from a sound source for both upwind and downwind positions.

Figure 1 shows some of the most important effects and limitations of ray tracing. This has implications for the audible sound and therefore these limitations are treated here briefly.

The person sketched at the upwind location does not receive any sound rays from the source. Accordingly, ray tracing predicts the so-called shadow zone to be a silent area where no sound from the source is present. Effects other than refraction, like diffraction, scattering due to turbulence and ground waves, are not included in Snell's law but cause some sound energy to be present in a shadow zone. The absence of any sound at all in the shadow zone, as predicted by ray tracing, is not accurate. To circumvent this limitation, a correction method based on the previously mentioned FFP is used. For typical linear sound speed profiles, a linear loss parameter (dB per meter into the shadow zone) is calculated. The parameter depends on the strength of the gradient of the effective sound speed and frequency. As a result, a linear empirical relation was found that is used to correct the ray tracing results:

$$
\begin{aligned}
& L_{d 1}=-0.0032-3.5 \cdot 10^{-5} f \\
& L_{d 2}=6.7 \frac{d c_{e f f}}{d z}+0.31 \\
& L_{d i f f}=L_{d 1} L_{d 2}\left(\left|x_{l}-x_{s z}\right|\right)
\end{aligned}
$$

where $x_{l}$ and $x_{s z}$ are the listener and the shadow zone positions (shown in Figure 1). $L_{\text {diff }}$ is the calculated correction in decibels based on two components $L_{d l}$ and $L_{d 2}$ which depend linearly on the frequency $f$ and the strength of the sound speed gradient from the ground up to the source height. The diffraction correction, resulting from Eq. (2), is added to the loss at the shadow zone boundary. This engineering method works well for linear sound speed gradients and was also checked against results of the FFP for a logarithmic wind speed profile. A typical result from this analysis is shown in Figure 2 for a source at an altitude of $400 \mathrm{~m}$ and a listener at $1.2 \mathrm{~m}$ height.
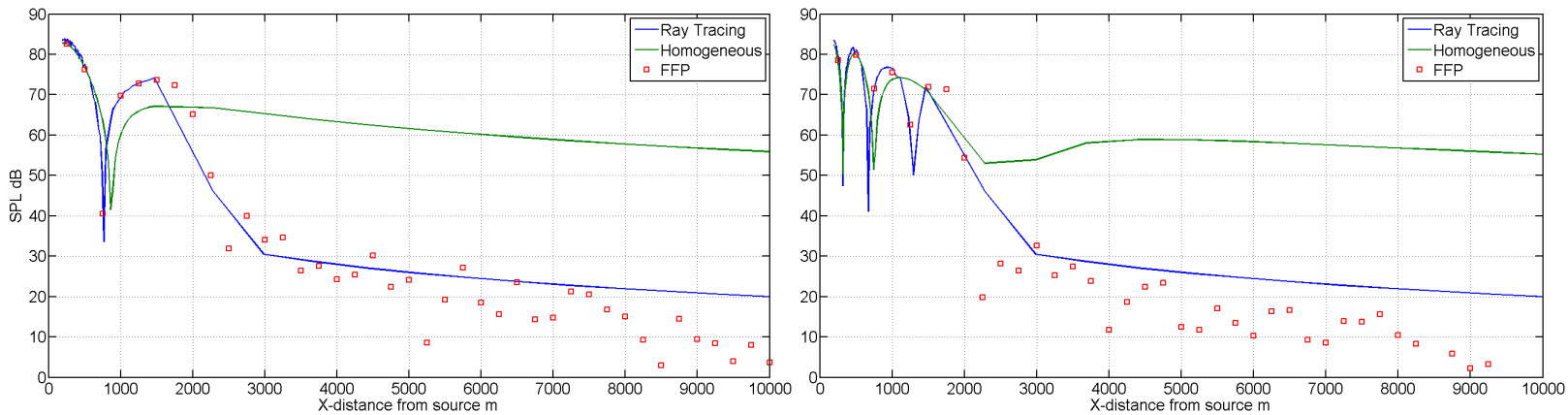

Figure 2 Typical results for the sound pressure level at a listener for $170 \mathrm{~Hz}$ (left) and $452 \mathrm{~Hz}$ (right) in a logarithmic wind profile. The ray tracing is augmented with the diffraction procedure and its results are comparable to the FFP. For reference, the homogeneous (no wind) solution is included. 
From Figure 2 it becomes clear that the method works relatively well for its intended purpose. The reduction in computation time is dramatic since no explicit FFP calculation is required during the course of the simulation. Since the correction method becomes unbounded with increasing distance into the shadow zone, a lower limit of this correction factor is used. This limit is set as $30 \mathrm{~dB}$ below the spherical spreading (homogeneous) solution.

In the case of downwind propagation, two other important effects are noticeable from Figure 1. The first one is a so-called "caustic." If the distance between two adjacent launched rays grows small, i.e., the rays become focused, an unbounded rise of the theoretical sound pressure level can occur. This is nonphysical and was therefore limited to $10 \mathrm{~dB}$ in excess off the spherical spreading solution. This limit was checked against FFP results and judged to be an adequate assumption. Another effect particular to the downwind direction is that more than a single ray pair (containing a direct and ground reflected path), or none at all, may reach the listener instead of always just a single pair for the homogeneous case. Sound reaching the listener might thus come from multiple directivity angles, each traveling through different atmospheric conditions. Therefore, for both upwind and downwind propagation, the emission angle at which the sound is synthesized is dependent on the weather conditions, in contrast to the synthesis for a straight-line path.

\section{Compact source}

Aircraft sound is composed out of many individual sources that propagate towards the listener. In the prior frameworks, the sources could be spatially distributed over the aircraft geometry, e.g., the jet noise comes from a different location than the landing gear noise. Such an approach was tractable since the path calculation was trivial. In case of the new framework, this would necessitate the ray tracing to be performed for every source location on the aircraft. This brute force approach requires an undesirable computational expense and therefore a slightly different approach is used. The alternative approach assumes the entire aircraft to be a compact sound source. As such, all sources follow the same propagation path, as found by ray tracing, and the source position is assumed to be some reference location, e.g., the center of gravity. The underlying assumption is that the difference in source locations on the aircraft does not affect the propagation path in such a way that different propagation characteristics are required. As a result, the total sound is composed by adding all individual sources that are propagated with a common ray tracing result. The only difference that is taken into account is the difference in travel time between individual sources. This difference is calculated based on straight-line path calculations. First the straight-line path travel time for each spatially distributed source is calculated and subtracted from the straight-line path travel time for the reference location. The resulting differences are then added to the common travel time of the curved ray path to obtain a unique travel time for each spatially distributed source. Using this approach, modeling of phase differences between (correlated) noise sources on the aircraft is still possible.

\section{Implications for the new framework}

Atmospheric propagation effects like curved paths are recognized to impact the audible results in several ways. To actually simulate the curved paths in the new framework, the following critical points need to be treated:

- The rays each originate from a different angle compared to the straight-line path case. This is important since most aircraft noise sources have directional radiation patterns. As a result, the noise that must be synthesized depends on the atmospheric conditions and the distance between the source and the listener.

- The number of paths is increased. In the case of the straight-line path assumption, there is only one ray pair containing a direct and ground reflected path. This is also true of the upwind condition shown in Figure 1, where the ground reflected ray is omitted for clarity. However, in the case of downwind propagation, there are two ray pairs for the condition shown in Figure 1. Consequently the number of paths increases from two to four. This requires additional bookkeeping and increases the simulation workload which is directly proportional to the number of paths.

- Along each path, the travel time of the sound is integrated to obtain the total travel time to reach the listener. The time rate of change of the time delay at the listener simulates the Doppler shift ${ }^{9}$ and must therefore be treated carefully.

- Along each path, the atmospheric absorption is accumulated as the sum of piecewise frequency dependent absorption per length. For the straight-line path with a stationary listener, this could be computed solely as a function of slant range (distance from source to listener).

- Along each path, the interaction of the ray with the ground must be accounted for by integrating the frequency dependent attenuation associated with each reflection. In realistic wind fields, the sound might reach a listener only through reflection off the ground. In Figure 1 this would require ground attenuation 
for the blue ray path but not the black ray path, as only the former reflects off the ground before it reaches the listener.

- As is seen from Figure 1, the angles at which the rays reach the listener vary per path. This has an impact on the propagated sound since the ground attenuation is a function of the incidence angle. Further, direction of the sound received by the listener is simulated using the receiver angle to determine the set of HRTFs used for binaural simulation, or the VBAP weights used for loudspeaker playback.

The propagation through a non-uniform atmosphere thus has an effect on every part of the simulation. As such, the computation has to start with calculation of the curved eigenray(s). The next section highlights some of the processing steps necessary in this new framework to treat these effects.

\section{Framework architecture}

Given the fact that the propagation is the driving calculation for the simulation framework, the prior approaches do not suffice, as they compute the straight-line path. Figure 3 shows the newly developed framework. It is convenient to describe the framework as consisting of three processing chains. The first chain is devoted to path definition and source noise synthesis. The second chain is responsible for applying the integrated time delay, atmospheric absorption, ground plane attenuation and spreading loss to propagate the sound to the ground. The third chain is responsible for rendering the propagated sound to the listener over headphones or a loudspeaker array. As previously indicated, this first implementation of the framework does not allow for real-time processing, but can be modified later to facilitate this. Each chain is discussed in further detail below.

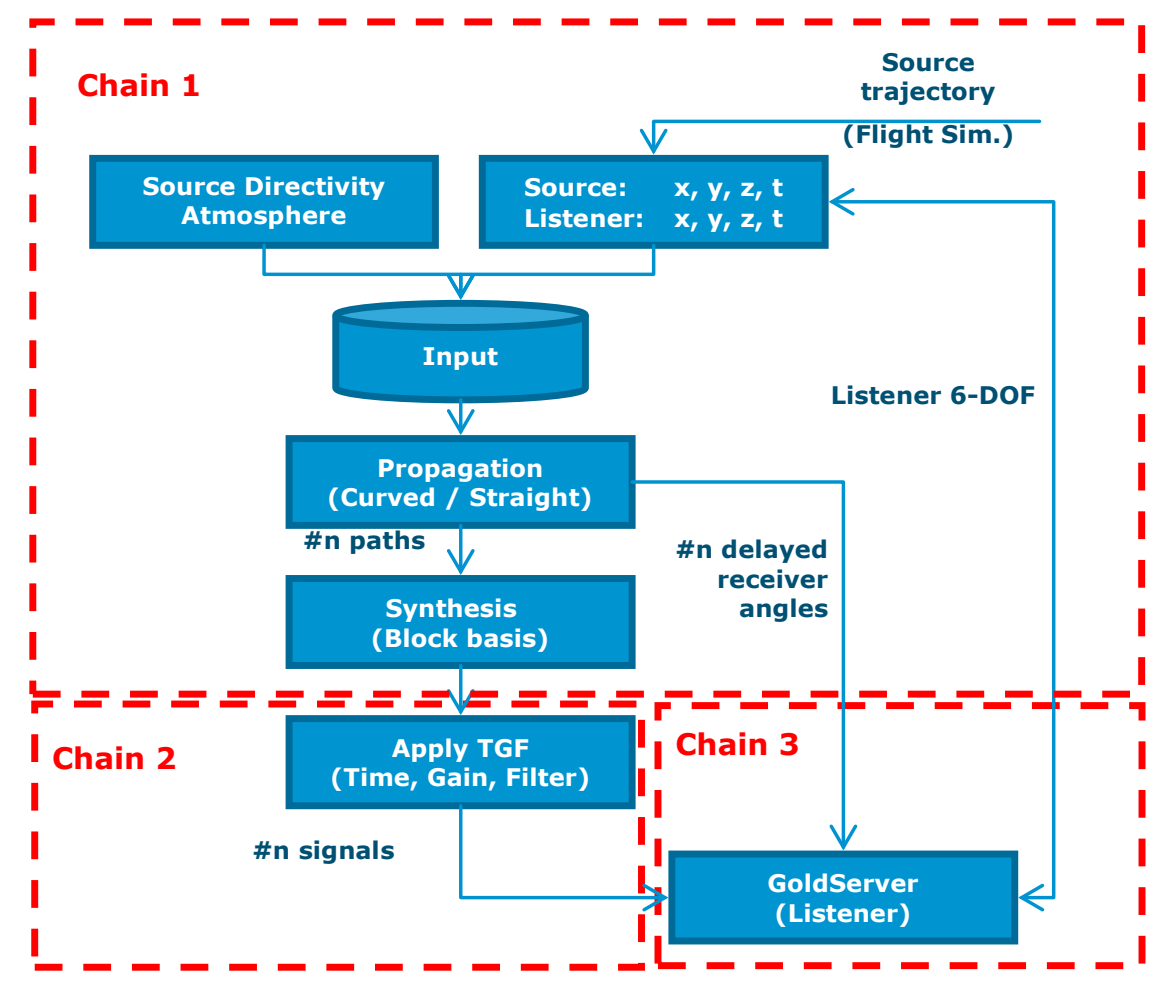

Figure 3 Flowchart of the new framework showing three processing chains.

In the first chain, information about the source and propagation medium, as well as the source and listener positions are required as input. Source noise synthesis may be based either on a priori computed source noise directivity patterns, as a function of discrete polar and azimuth angles (spheres or hemispheres), or on real-time computed directivity patterns using empirically based models. In either case, the directivity is a function of the operating condition and can be expressed in either the frequency or time domain. Calculation of realistic atmospheric input is not trivial and therefore measurements are preferred for input. ${ }^{22}$ Note that scattering due to atmospheric turbulence is a time dependent characteristic not presently included in the simulation. Ground impedance model parameters, e.g., flow resistivity, are also required as input. 
In the initial, non-real-time, version of the framework, the source and listener trajectories are specified as part of the input. In a future real-time implementation, the aircraft position could come from a flight simulator and the listener position from a tracking sensor (as shown). Under either scenario, once the input data is specified, a loop over simulation time is started in which the curved path analysis and synthesis are performed. For every time increment, the aircraft and listener positions are interpolated. It should be noted that the source and listener trajectories are also required for visual scene generation in the virtual reality environment (not explicitly shown in Figure 3). Within the virtual reality environment, the real-time listener orientation is required to position the virtual source according to the receiver angle obtained from the path analysis. The operation is performed within the third processing chain.

Note that some limitations on the listener movement are presently required. The path calculation is performed at each time increment based on the instantaneous source and listener positions, i.e., at the emission time. Since the travel time may be many seconds, it must be assumed that the listener does not translate sufficiently far during that time so as to necessitate a new path calculation. Further, although the ground impedance can vary according to location, the terrain in which the listener moves must be flat to preclude the possibility of the listener moving behind an obstacle, e.g., mountain or building, and partially shield the incoming sound. Diffraction around obstacles is presently not incorporated.

From the ray tracing analysis, the number of possible paths and their corresponding emission and receiver angles are computed. As indicated above, the receiver angles are used in the third rendering chain to position the source in the virtual reality environment. The emission angles are used to synthesize the instantaneous pressure time histories (one per path) at the moving source, using the source directivity as described above. In this respect, the new framework is consistent with the earlier CNoTE implementation. The synthesis methodology is dependent on the source type. Previous work by the authors focused on broadband synthesis ${ }^{9,10}$ (e.g., jet noise), tonal synthesis ${ }^{12}$ (e.g., fan noise), and time domain synthesis ${ }^{11}$ (e.g., rotary wing noise).

Concurrent with the synthesis operation, the integration of time delay, atmospheric and ground plane absorption, and spreading loss is performed per path. The absorption and spreading loss are expressed as linear phase Finite Impulse Response (FIR) filters, as described in Section II.E. This information together with the synthesized pressure time histories is passed to the second chain for propagation processing. In the present implementation, the time loop is incremented through the course of the full trajectory until the entire pressure time histories of the moving sources are synthesized along with the time histories of integrated time delays and filters.

In the second chain, the synthesized sounds at the moving sources are propagated to produce pseudo-recordings at the listener position. The framework was built in Matlab and a dedicated off-line DSP engine was programmed to apply the integrated time delay and filter to the synthesized sound source. In the future real-time framework, this operation will be executed on the GoldServer ${ }^{\mathrm{TM}}$.

In the final processing chain, the sounds are rendered to a tracked listener using the Goldserver ${ }^{\mathrm{TM}}$ which works together with a real-time graphics server to produce the virtual reality environment. Together the audio and graphics servers complete the simulation of the flyover event including realistic positioned audio and visual cues. The positional audio is achieved using binaural simulation with HRTF filtering for playback over headphones, or using VBAP for playback over a speaker array. In the present implementation, the time delayed receiver angles are used to position the virtual sources. In this fashion, the rendering chain is performed in the same manner as if the propagated source at the listener position were an actual microphone recording at the simulated listener height. ${ }^{9}$

\section{E. Applying atmospheric propagation through DSP}

The propagation characteristics, e.g., travel time, spreading losses and absorption, are calculated with the help of ray tracing. Applying these calculated characteristics is done by processing the synthesized sound with delay, filtering and gain operations. At each processing increment of the first chain, the time delay is calculated as the integrated time along the curved path. The time delay decreases as the aircraft nears the listener and increases as the aircraft retreats. The rate in which the time delay decreases and increases is proportional to the Doppler shift as it effectively compresses and stretches the synthesized source pressure time history. The total delay is applied on a sample-by-sample basis at a regular audio sampling rate much greater than the time increment used to calculate the path. Therefore, the delay must be interpolated at the sample level and fractional delay lines must be used. ${ }^{9}$

In the case of spherical spreading of sound, i.e., straight-line propagation path, the spreading losses are frequency independent and the gain is readily calculated from the geometry. The spreading losses for curved paths depend on the spatial density of the sound rays near the listener. In case of ray focusing, the rays are close together, so the losses are less than spherical spreading. The opposite holds for defocusing, where the loss is greater than spherical spreading. There is no frequency dependency in the focusing modeling approach, but the shadow zone correction 
depends on the frequency, see Eq. (2). As a result, the total spreading loss is frequency dependent for the curved path. Applying a gain to the source sound does not suffice in that case and a filter is necessary.

The filtering operation treats three separate effects: the atmospheric absorption, the spreading losses and the ground reflection. Atmospheric absorption is a frequency dependent loss that depends on humidity and temperature along the ray path. Spreading losses, frequency dependent or not, are added to the atmospheric absorption to find an overall frequency dependent loss. The FIR filter taps are computed by taking an Inverse Fast Fourier Transform (IFFT) of the desired (magnitude only) frequency response. The filtering operation is performed in an overlap-add fashion $^{23}$ which allows the filtered output to vary smoothly with changes to the filter during the course of a flyover. If the ray reaches the listener after being reflected off the ground, the ground impedance must additionally be taken into account. The ground impedance is regarded as a complex valued transfer function. ${ }^{24}$ The combined atmospheric and spreading losses are multiplied with the ground impedance transfer function in the frequency domain, i.e. a convolution operation, and inverse transformed to obtain the required FIR filter taps. The resulting filter is applied to simulate the ground reflected path after being delayed by a small additional travel time due to the ray path length difference. This small delay effectively causes the well-known ground interference pattern, referred to as a comb filter in the literature ${ }^{23}$, after mixing the direct and ground reflected propagated signals.

\section{Results}

A full flyover simulation using the newly developed framework is demonstrated. The aircraft source was comprised of jet noise ${ }^{13}$ combined with tonal components of fan noise ${ }^{12}$. The jet noise prediction was based upon a CF6-80C2 engine from a Boeing 747-400, whereas the fan noise prediction was based upon the Honeywell Tech977 engine. ${ }^{25}$ Because the simulation is prediction-based, alternative configurations are possible. Each noise component is synthesized independently and combined prior to propagation. The aircraft itself is flying a straight and level trajectory from $x=6000$ to $-6000 \mathrm{~m}$, at an altitude of $152 \mathrm{~m}(500 \mathrm{ft}$.), with a speed of $100 \mathrm{~m} / \mathrm{s}$. Both the engine fan and jet noise components are simulated at $87 \% \mathrm{~N} 1$.

The listener is moving perpendicularly away from the ground track of the aircraft at a walking pace $(5 \mathrm{~km} / \mathrm{h})$. As a consequence the aircraft does not fly directly over the listener. The ground surface specified had a variable effective flow resistivity and the ground impedance is calculated with the Delaney \& Bazley mode ${ }^{24}$. As the listener was allowed to walk through the virtual environment, the impedance changed slowly. The varying impedance is categorized, for the entire simulation, as "rough roadside dirt."26

Researchers often apply a boundary layer model that is valid close to the ground, which yields logarithmic wind and temperature profiles for acoustic propagation problems. The most well-known of the boundary layer models are formed by the Businger-Dyer equations. ${ }^{22}$ These equations exclude the possibilities of wind speed inversions and changing wind directions. These limitations are circumvented by using measurements. The atmosphere specified was measured at Wallops field (VA) on the $10^{\text {th }}$ of October 2011 at noon. The measurement is accessible through a University of Wyoming internet site ${ }^{27}$ and is depicted in Figure 4.
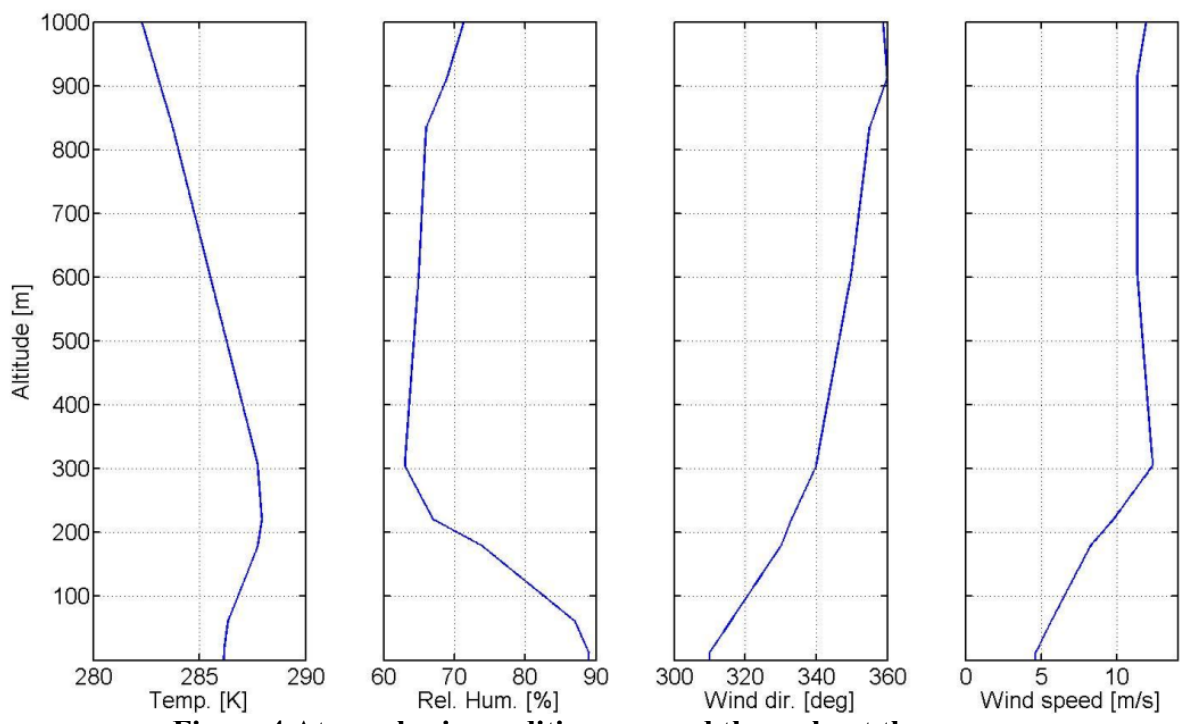

Figure 4 Atmospheric conditions as used throughout the paper 
The depicted atmosphere has a temperature inversion around $200 \mathrm{~m}$. An inversion is defined here as an altitude where the gradient of the quantity changes sign. In this particular atmosphere, the temperature increases from the ground level up until $200 \mathrm{~m}$ where it starts to decrease. Since the simulated aircraft flyover occurs at $152 \mathrm{~m}$, it is below the temperature inversion. The relative humidity and temperature are important in the calculation of the atmospheric absorption. ${ }^{28}$ The wind direction changed slightly from a North-East wind at the ground to a Northern wind at higher altitudes. The corresponding wind speed increased to a maximum of roughly $13 \mathrm{~m} / \mathrm{s}$ at $300 \mathrm{~m}$. The aircraft is flying on a North bound track, i.e., going towards the North thus moving from an upwind to a downwind position relative to the listener. The corresponding sound speed profiles were calculated and are shown in Figure 5. Note that the straight-line path results were calculated for a non-uniform atmosphere for accurate accumulation of the total atmospheric absorption, but wind effects were ignored.

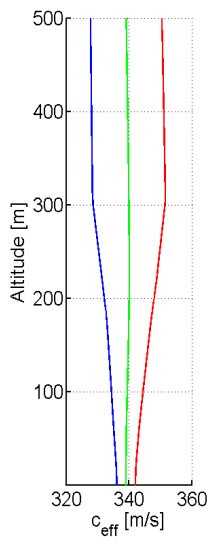

(a)

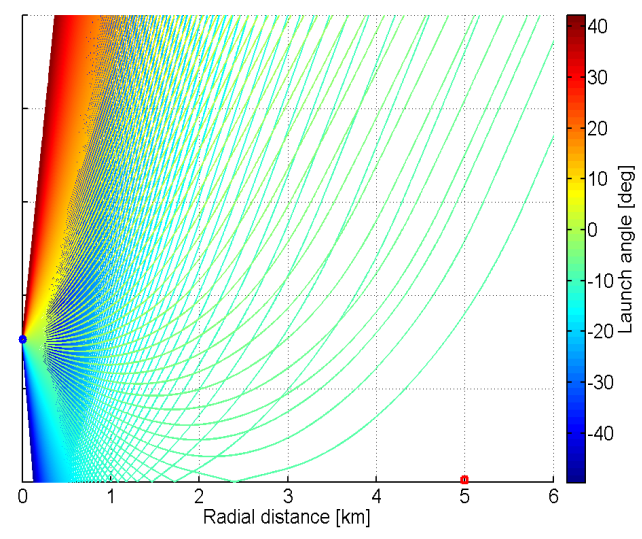

(b)

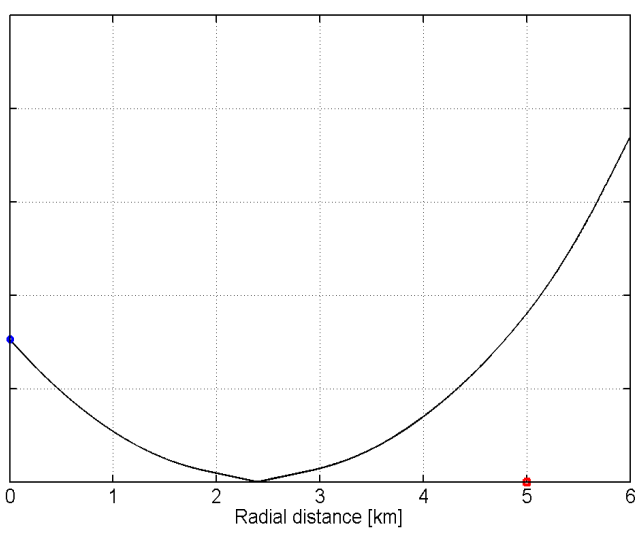

(c)

Figure 5a shows three sound speed profiles: the upwind (blue), downwind (red) and temperature only (green). Figure 5b shows the general ray tracing result for the upwind sound speed profile; the blue dot is the source and the red square the listener. Figure 5c shows the resulting eigenray, i.e., the ray closest to the listener.

\section{A. Ray tracing results}

The ray tracing algorithm launches sound rays from the source position at many initial launching angles. Only the ray that reaches the listener is of interest for further processing as it contains the path that the synthesized sound will follow. This particular ray is called an eigenray. Finding the eigenray is exercised in an iterative manner by zooming in on the closest ray, and re-launching a new cluster of rays in a new direction around the last closest ray. After a few iterations an eigenray is found with the desired accuracy. Not only is the angular resolution refined by this iterative procedure, the time step of the integration is reduced as well. As a result, the first iterations are done computationally fast to establish a direction of where to find the eigenray. Further temporal and angular refinement gives the desired accuracy, albeit with increasing computational expense. In Figure 5c, the eigenray does not come near the listener because of the upwind conditions (Figure 5a, blue line). This means that the listener is in a shadow zone and the diffraction correction, described earlier, is used. Note that the curvature of the rays depends on the gradient of the speed of sound profile. Looking back to Figure 5a, it becomes clear that the sound speed profile based on the temperature only (green line) does not show gradients as large as the up- or downwind case. Atmospheric refractive effects are in this case primarily caused by wind gradients rather than temperature gradients.

As the aircraft flies along the trajectory, the speed of sound profile changes according to Eq. (1). The ray tracing is recalculated to obtain the eigenray(s) for each new aircraft position relative to the listener. Figure 6 shows snapshots of the eigenray(s) as the aircraft is flying through the atmosphere along its trajectory. The distance indicated on the abscissa is the relative distance between the projected source and listener. That is, the listener moves slowly, but the main change is due to the aircraft moving left to right. The first snapshot of Figure 6 corresponds to the conditions found in Figure 5. 


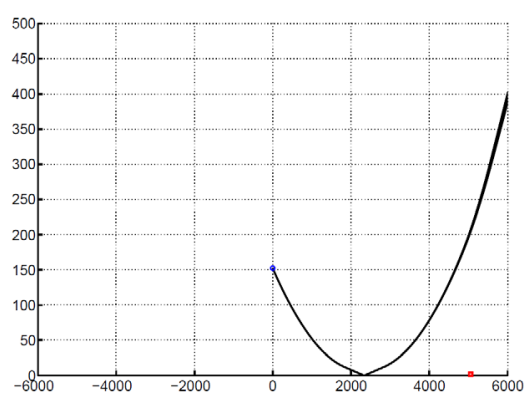

$\mathrm{t}=10 \mathrm{~s}$.

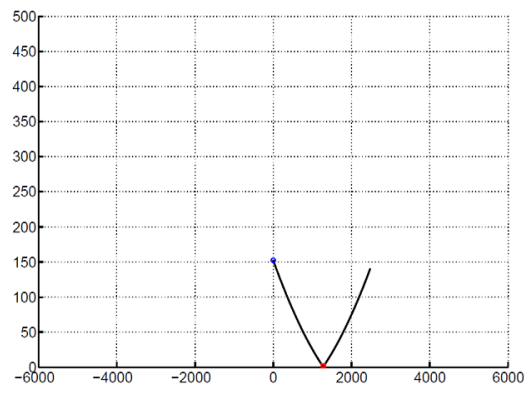

$\mathrm{t}=46 \mathrm{~s}$.

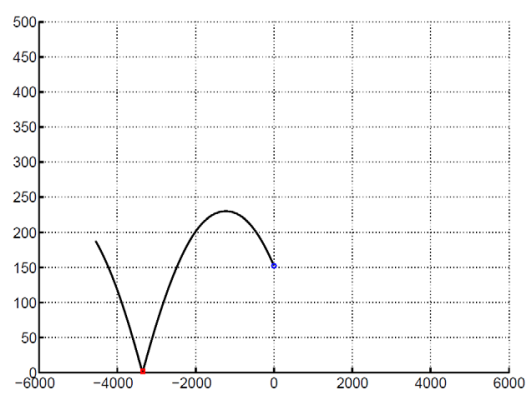

$\mathrm{t}=90 \mathrm{~s}$.

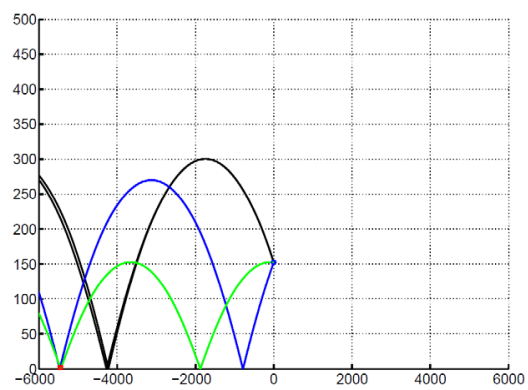

$\mathrm{t}=110 \mathrm{~s}$.

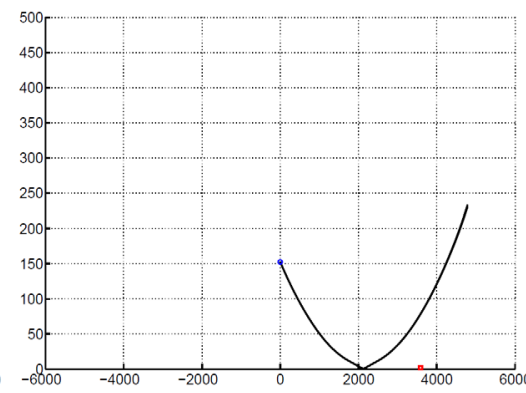

$\mathrm{t}=24 \mathrm{~s}$.

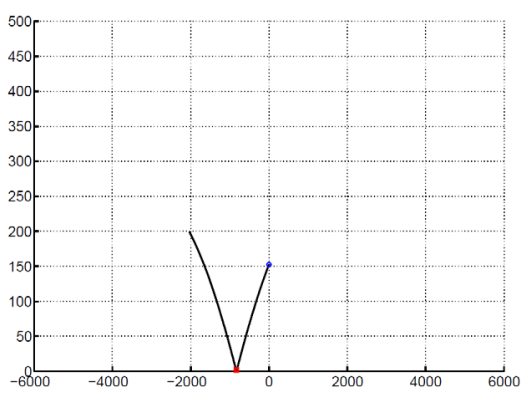

$t=66 s$.

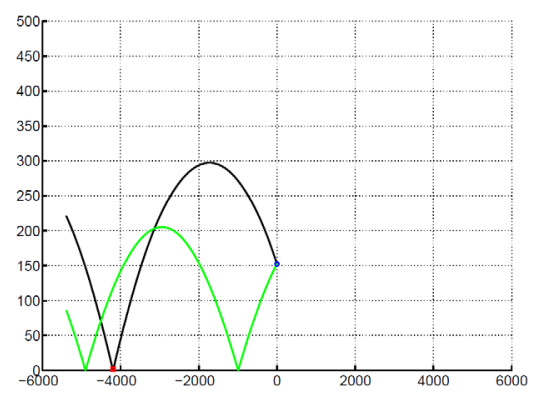

$\mathrm{t}=97 \mathrm{~s}$

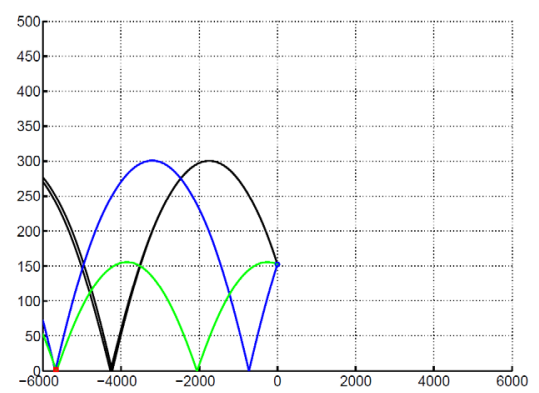

$\mathrm{t}=112 \mathrm{~s}$.

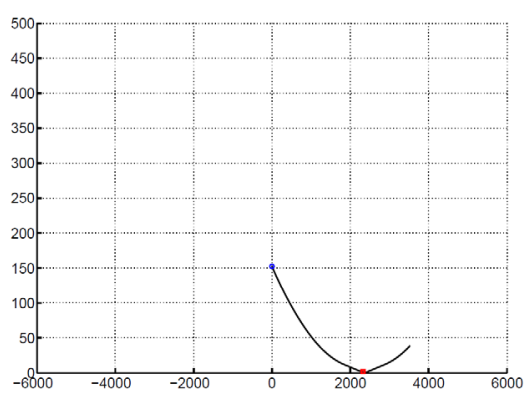

$t=36 s$.

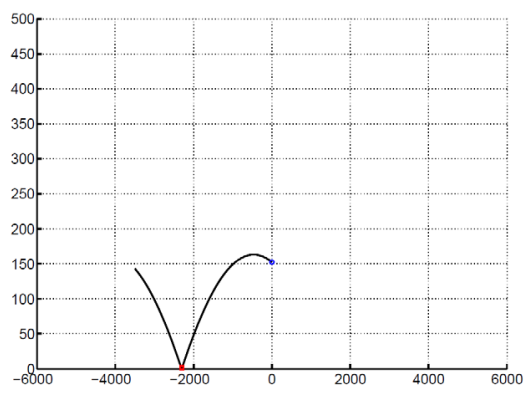

$\mathrm{t}=80 \mathrm{~s}$.

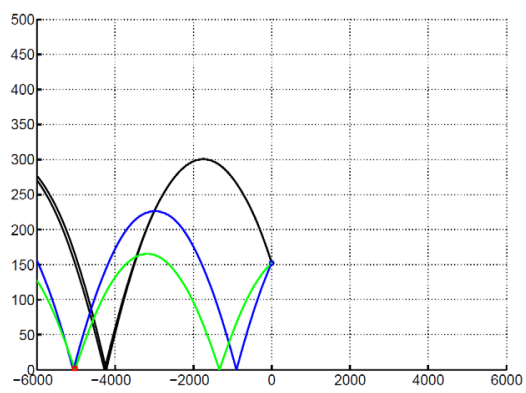

$\mathrm{t}=106 \mathrm{~s}$.

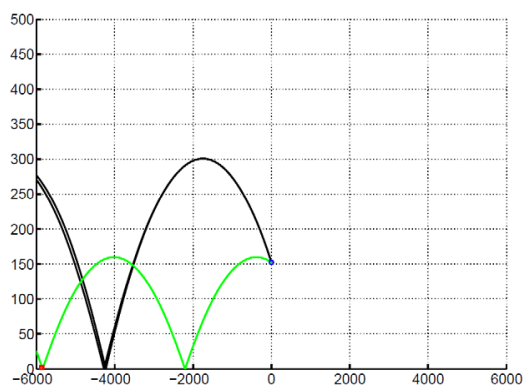

$\mathrm{t}=114 \mathrm{~s}$.

Figure 6 Snapshots depicting the eigenray(s) from different points along the aircraft trajectory are taken. The time shown is the emission time, i.e., the time at the source. From 0-58s, the aircraft is upwind and from 58-114s, the aircraft is downwind. The black line is the $1^{\text {st }}$ eigenray, the green line is the $2^{\text {nd }}$ eigenray and the blue line is the $3^{\text {rd }}$ eigenray. 
The emission angle of the sound can be calculated for use in noise synthesis from the launch angle of the eigenray(s). The emission angle is the angle used in the calculation of the source directivity pattern. In case of the Stone jet noise model $^{29}$ or the Heidmann fan model ${ }^{30,31}$, the source is assumed to be axisymmetric, i.e., no azimuthal dependency. In this work, the small fan update to the Heidmann fan model, as implemented in ANOPP ${ }^{15}$ is used. The emission angle can differ from the launching angle in two ways. First, the engine can be tilted upward or even change its orientation during flight, e.g., like a Bell Boeing Osprey tilt-rotor. Second, the source directivity models may be limited to a range of emission angles, as discussed below. Taking these considerations into account, the emission angle is calculated and is shown in Figure 7 for a fixed upward tilt angle of 1 degree.

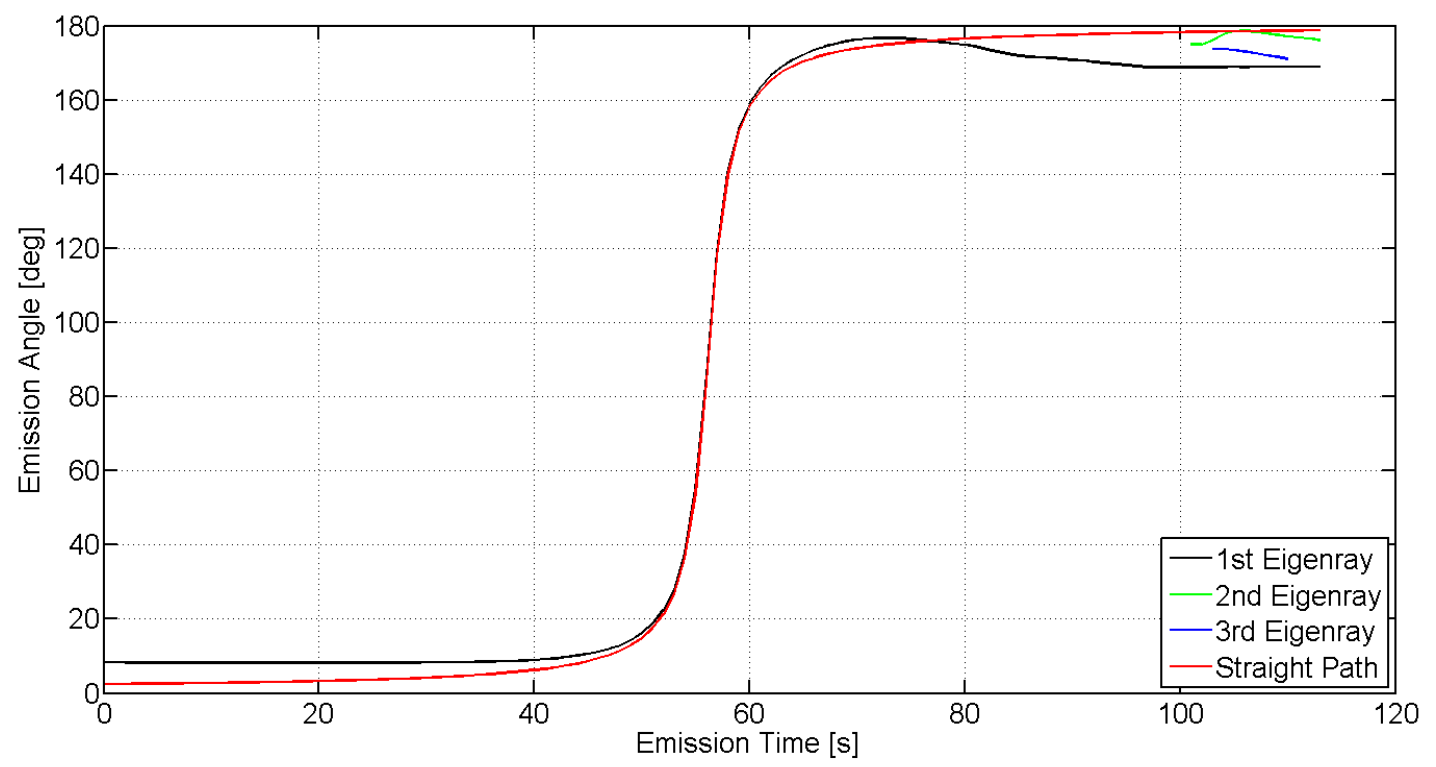

Figure 7 As a result of the curved eigenrays, the emission angle has changed from the straight path assumption. The effect is the largest at the start and end of the simulation.

From 0-36 s there is a visible difference between the straight and curved path emission angles in Figure 7. This is because the aircraft is in a shadow zone; a propagation phenomena that is not be captured by the straight path approach. The $1^{\text {st }}$ eigenray has a constant emission angle from the limiting ray, i.e., the ray that forms the boundary between the "illuminated" zone and the shadow zone. This effect is also noted in the snapshots of Figure 6, during the first $36 \mathrm{~s}$ where the eigenray remains roughly the same. Due to bending of the rays, no ray reaches the listener and therefore the closest ray and its characteristics are used.

When the aircraft emerges from the shadow zone the difference between the emission angle of the $1^{\text {st }}$ eigenray and the straight path quickly diminishes and the straight path result is essentially recovered. In other words, the rays nearly follow the straight line ray paths. This is also noticeable in the snapshots of Figure 6 where the curvature becomes larger for larger distances between the source and listener.

From the snapshots it also becomes apparent that the launching angle changes significantly between the upwind and downwind positions. Referring to snapshots at 10 and $80 \mathrm{~s}$, the relative positions between the listener and source are similar, although the former is upwind and the latter downwind. In the first case the rays are launched downward and are curved upwards, whereas in the latter case the rays are launched upwards and are bent downwards.

At around $75 \mathrm{~s}$, the first eigenray is launched horizontally. The emission angle shows a maximum at that position, but is less than 180 degrees because the source is tilted and the listener is down and to the side. After about $75 \mathrm{~s}$, eigenrays are launched upward. Note however that since the directivity pattern is axisymmetric, it is mirrored about 180 degrees. As a matter of bookkeeping, directivity angles in excess of 180 degrees are indicated at their mirrored value. Consequently, the emission angles shown in Figure 7 begin to decrease after $75 \mathrm{~s}$ as the launch angle becomes more upward, see Figure 6.

After $102 \mathrm{~s}$, there are multiple eigenrays that reach the observer, each with a unique emission angle and ray path. This is in contrast with the straight-line path result for which there can only be one ray. In the case of multiple eigenrays due to atmospheric conditions, the straight path assumption is not valid. As such, comparing the emission angle of the straight path to the $2^{\text {nd }}$ and $3^{\text {rd }}$ eigenray is not valid. 
The receiver angle is also affected by the curved rays. A change in receiver angle affects the ground plane reflection in two ways; the wave reflection coefficient is dependent on the incidence angle and differences in path length between the direct and ground reflected rays (relative to the straight line path case) cause a different ground interference pattern. A change in receiver angle from the straight-path also affects the perception of the source location by the listener. Figure 8 shows the receiver angle for the curved paths and the straight path.

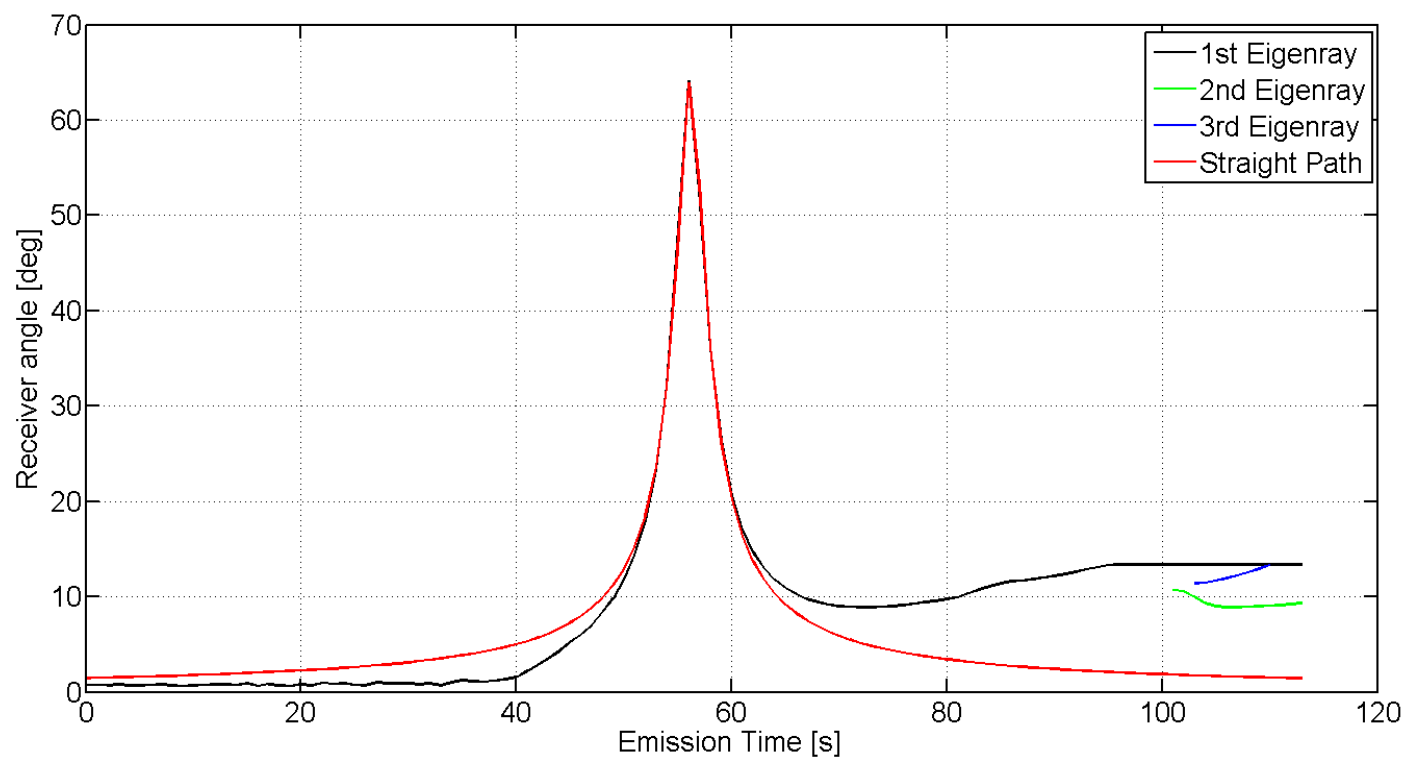

Figure 8 The receiver angle as used for the ground reflection coefficient calculation and the path length difference between the direct (eigen)ray and the ground reflected ray.

From Figure 8 it is clear that the receiver angle is symmetric for the straight path assumption whereas the receiver angle is asymmetric for the curved ray paths. The receiver angle does not reach 90 degrees as the listener is moving, in this case at a walking pace to the side of the aircraft ground track. The first eigenray receiver angle is, as for the emission angle, constant during the first $36 \mathrm{~s}$ due to the fact that the limiting ray is used. A few small wrinkles are visible and will be explained in the following section addressing total loss. After emerging from the shadow zone at around $36 \mathrm{~s}$, the difference between the straight path and the first eigenray quickly diminishes. As the aircraft flies over $(\sim 57 \mathrm{~s})$ the receiver angles are similar as the rays do not exhibit much curvature. At around $65 \mathrm{~s}$, the receiver angle of the $1^{\text {st }}$ eigenray does not decrease further and is relatively constant up until $80 \mathrm{~s}$, after which it starts to increase again. This means that the sound at the listener is coming in from a steeper angle than expected from a straight path assumption. After roughly $97 \mathrm{~s}$ the receiver angle becomes constant for the first eigenray. This can be explained by the snapshots where it is seen that the first eigenray can no longer reach the listener. If a ray goes over an altitude of $300 \mathrm{~m}$ along its trajectory, the ray is refracted upwards by the wind. This is due to the decreasing sound speed above this altitude in the downwind condition (red line in Figure 5a). As such, the eigenray cannot reach the listener and a constant angle of the limiting case is used together with the diffraction correction to simulate the sound emanating from the nearest ray position, per Eq. (2). The eigenray solution is retained as the aircraft retreats because the same eigenray might again reach the listener at some greater distance, see for example the $2^{\text {nd }}$ eigenray at $114 \mathrm{~s}$.

Like the emission angle (see Figure 7), the receiver angle of the $3^{\text {rd }}$ eigenray grows towards the angle of the $1^{\text {st }}$ eigenray. Looking back to the snapshots it is seen that the corresponding ray paths reach the same maximum altitude of $300 \mathrm{~m}$. Any ray going over this altitude is refracted upwards and thus outside the range of interest for this simulation. Since both eigenrays follow similarly curved, albeit offset, trajectories, similar receiver angles are found since the rays follow identical arcs.

The associated losses per ray, as used in the filters, are depicted in Figure 9 for three different frequencies. Note that the shown losses are generally compiled from the spreading loss and the atmospheric absorption. In case the eigenray has reflected off the ground before reaching the listener, e.g., eigenray 2 and 3, the ground attenuation is included as well. 

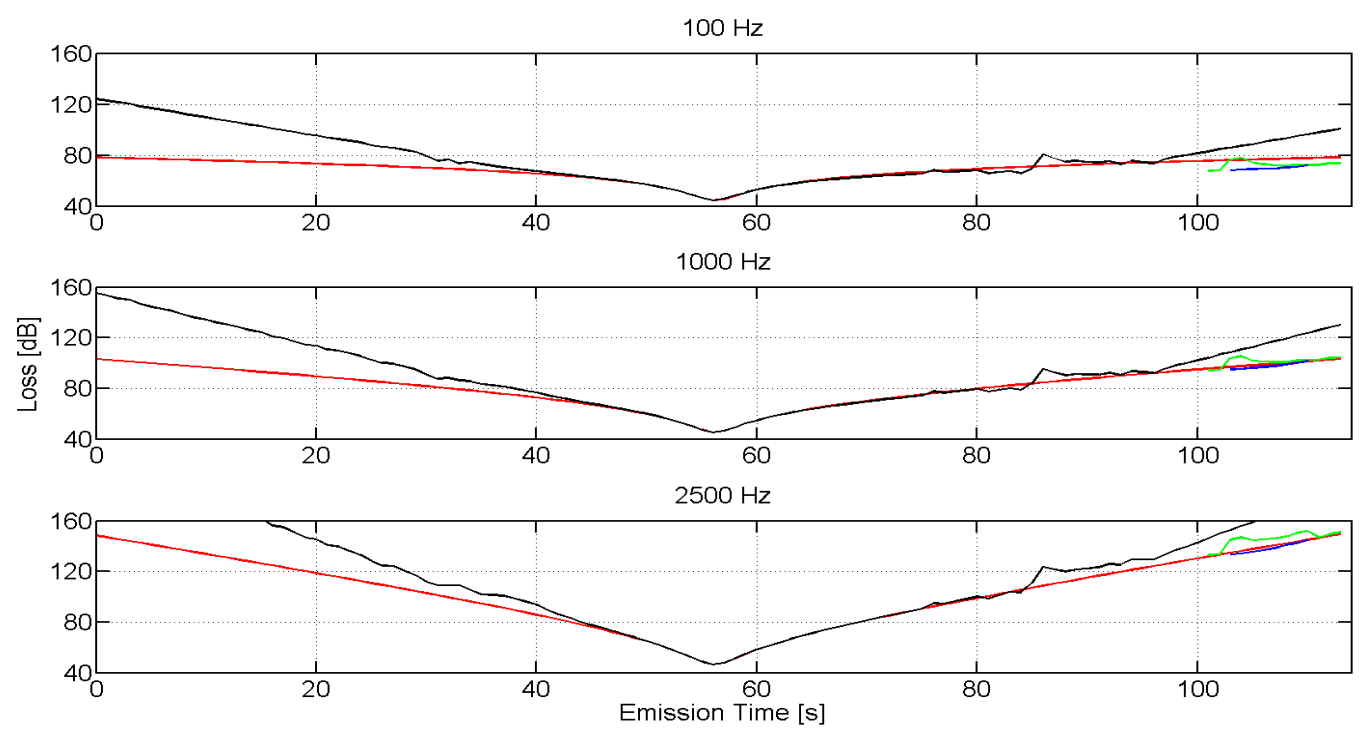

Figure 9 The total loss as used to obtain the filter characteristics for three typical frequencies. The red line is the straight path result, the black line is the $1^{\text {st }}$ eigenray, the green line is the $2^{\text {nd }}$ eigenray and the blue line is the $3^{\text {rd }}$ eigenray.

As with the emission- and receiver-angle, the straight path (red line) is symmetric whereas the curved path results are not. The shadow zone associated with the upwind propagation is present from 0-36 s. Clearly the losses are much larger compared to the straight path result due to the shadow zone and these losses increase with increasing frequency. At around $36 \mathrm{~s}$ the shadow zone ceases to exist as the aircraft comes closer. This is noticeable by small wrinkles in the loss lines for the first eigenray. Note that the wrinkles become more pronounced for the $2500 \mathrm{~Hz}$ result even before the $30 \mathrm{~s}$ mark. This is due to the fact that the limiting ray, i.e., the ray dividing the illuminated and shadow zone, is very sensitive to launch angle. A small deviation in launching angle due to a moving aircraft or listener, changes the sound speed profile as in Eq. (1) and results in a small difference in the shadow zone position and corresponding spreading loss. Since the losses are well over $80 \mathrm{~dB}$, or even closer to $100 \mathrm{~dB}$ for the $2500 \mathrm{~Hz}$ solution, small wrinkles have no audible effect on an already highly attenuated signal.

As the aircraft emerges from the shadow zone and comes closer to the listener, the rays become less curved. As a result the differences in total loss between the straight path and the $1^{\text {st }}$ eigenray become smaller and diminish when the aircraft is flying closest. From $75 \mathrm{~s}$ onwards, the $1^{\text {st }}$ eigenray loss line starts to show some wrinkles again. Looking back to the snapshots in Figure 6 it is noticed that the launching angle has crossed the horizontal at this position. At that point the ray is launched at a very shallow angle from the source. At shallow launching angles, rays are susceptible to small changes in the sound speed profile. As the aircraft is moving, and the sound speed profile changes, eigenrays reaching the listener launched at a shallow angle show the small changes in sound speed profile in their overall loss as small wrinkles. Around $85 \mathrm{~s}$, this effect becomes more prominent as the rays reach an altitude of $220 \mathrm{~m}$. At that point, the wind direction changes slightly causing, yet again, a tiny irregularity in the sound speed profile. Rays travelling through this irregularity at a shallow angle are affected, i.e. curved more towards the ground. Rays reaching this altitude at a steeper angle are relatively unaffected and curve at a more gradual rate towards the ground. Figure 10 shows this effect with a snapshot of some rays launched from the source at an emission time of $85 \mathrm{~s}$. 


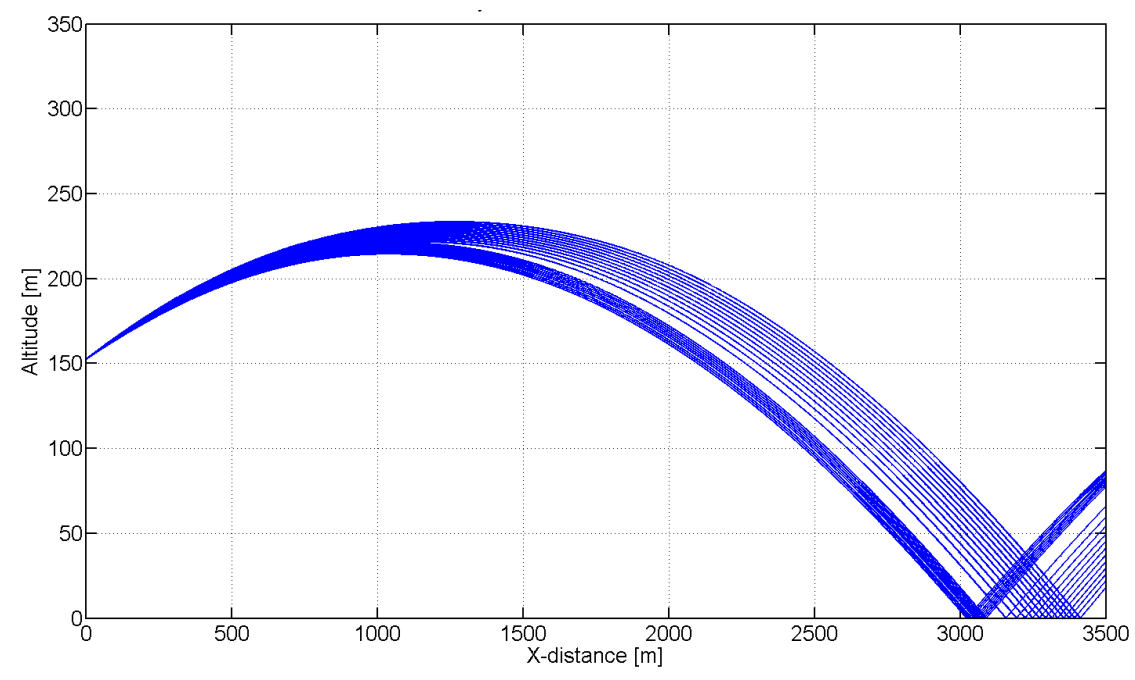

Figure 10 At an emission time of $85 \mathrm{~s}$, rays launched from the source experience a very shallow angle at a downwind range of around $1250 \mathrm{~m}$. Note the difference in ray density before and after $3050 \mathrm{~m}$.

Near 3000-3050 m the rays are close together, i.e., they are focused as shown in Figure 10. Further downwind, the rays reach the ground but are spread farther apart, i.e., they are defocused. Close observation of Figure 9 shows that before $85 \mathrm{~s}$ the loss is lower compared to the straight path result. After a few seconds the loss becomes higher again. This is due to the focusing and defocusing of the rays originating as a combination of a small irregularity in the sound speed profile and the shallow angle at which the ray reaches the irregularity. The consequence of this is audible as a modulation of the overall sound level.

Around $97 \mathrm{~s}$, the $1^{\text {st }}$ eigenray reaches its limiting altitude of $300 \mathrm{~m}$, above which upward refraction negates the possibility of that ray reaching the listener. From that time on, the diffraction correction is applied as the shadow zone moves over the listener. This condition remains in effect up until $102 \mathrm{~s}$, at which point the $2^{\text {nd }}$ eigenray becomes feasible and propagates sound from the source towards the listener. From $104 \mathrm{~s}$ onwards, the $3^{\text {rd }}$ eigenray is feasible as well. Both the $2^{\text {nd }}$ and the $3^{\text {rd }}$ eigenray are focused and lead to a smaller spreading loss compared to the straight path result. However, due to the curvature, the ray path is extended and the rays experience more absorption. The remaining sound energy is further attenuated by the ground reflection along the ray paths. These two effects balance the effect of focusing. The low frequency results are not influenced as much by the attenuation and therefore show the distinct effect of focusing, a (slightly) lower total loss than the straight path. Focusing effects are thus expected to only have a noticeable effect on the low frequency sound emanating from an aircraft.

\section{B. Synthesis results}

Having all the propagation characteristics at hand, it is possible to apply them to the synthesized source sound and render the sound at the listener. The resulting pseudo-recordings are presented as spectrograms showing the distribution of sound energy for the aircraft flyover. Figure 11 shows the spectrograms for the straight path propagation (top figure) and the curved path propagation (bottom figure), where the time indicated is the receiver time, and the maximum frequency shown is limited to $10 \mathrm{kHz}$ for clarity. The forward and aft radiated fan tones are clearly visible and were synthesized at the blade passage frequency (BPF) and three harmonics of the BPF. Also visible are "buzz-saw" tones which are present at multiples of the shaft speed at high power settings. The effect of Doppler shift is clearly seen in the tones. At the closest location, the Doppler shift factor is a minimum and the tones are close to the BPF frequency $(3258 \mathrm{~Hz})$ and its harmonics.

The indentations in the spectrogram indicate the comb filtering effect caused by the ground interference. The comb filtering pattern for the straight path is, as expected, consistent with earlier work. ${ }^{9}$ The ground interference pattern associated with the curved path is markedly changed. The indentations are curved as a result of the different receiver angles demonstrated in Figure 8. The asymmetry of the receiver angles manifests itself in the spectrogram and results in an upward turned interference pattern for upwind conditions and downward turned pattern for downwind conditions.

Because the synthesis was started at an upwind distance of $6000 \mathrm{~m}$, this resulted in a roughly $17 \mathrm{~s}$ period of dead time before the sound is audible. This is an artifact of the manner in which the simulation began. For the straight path, this causes the rather abrupt presence of the sound. For the curved path, the upwind conditions indicate the 
presence of the shadow zone which attenuates the sound until it emerges at a receiver time of roughly $45 \mathrm{~s}$. As a result of the shadow zone, the first BPF is audible at an earlier stage $(\sim 35 \mathrm{~s})$ for the straight path propagation case compared to the curved path propagation a few seconds later.
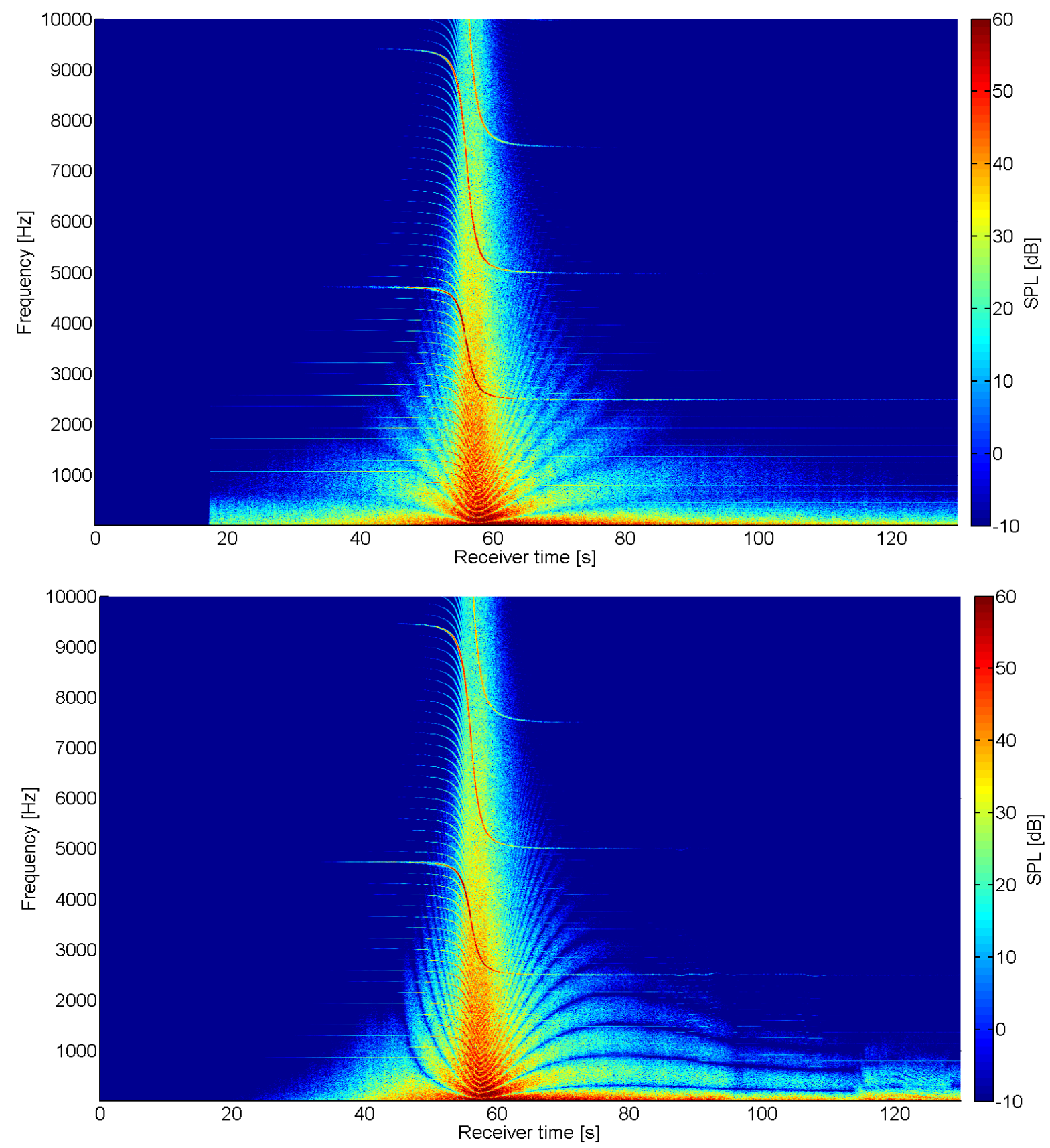

Figure 11 The spectrograms for the straight path assumption (top) and the curved path assumption (bottom).

Besides the changes in the ground interference pattern, the increasing loss at around $85 \mathrm{~s}$ emission time is due to entrance into the shadow zone of the $1^{\text {st }}$ eigenray (see Figure 6), which is clearly visible in the spectrogram of the curved case at a receiver time of $\sim 98 \mathrm{~s}$. From that point up until $117 \mathrm{~s}$ the audible sound is decreased as there are no rays reaching the listener directly despite the downwind conditions. At $117 \mathrm{~s}$, the $2^{\text {nd }}$ and $3^{\text {rd }}$ eigenray paths start to propagate sound towards the listener and increase the noise level. During the period from 85-117 s, only low frequency noise is present. After $117 \mathrm{~s}$, the ground interference pattern is obscured since multiple paths with different ground interference patterns interact to fill up the indentations. 
The resulting $\mathrm{L}_{\mathrm{A}, \max }$ and Sound Exposure Level (SEL) values, integrated over the entire flyover, are shown in Table 1.

Table 1 Integrated noise metrics for the straight path and curved path result

\begin{tabular}{|c|c|c|}
\hline Metric & Straight path - dB(A) & Curved path - dB(A) \\
\hline $\mathrm{L}_{\mathrm{A}, \max }$ & 78.90 & 78.45 \\
\hline $\mathrm{SEL}$ & 84.24 & 84.21 \\
\hline
\end{tabular}

From Table 1 it becomes clear that the difference between the straight and curved path approach is small. This is due to the fact that the $\mathrm{L}_{\mathrm{A} \text {,max }}$ value is typically measured when the distance between the aircraft and listener is the smallest. At that point the eigenrays are relatively straight and, as a result, return the same results as using the straight path assumption. The difference in SEL is, as a result, minute since it is dominated by the higher values of $\mathrm{L}_{\mathrm{A}}$. This effectively shows that weather dependent effects are not reflected in typical metrics in case of an aircraft flyover for the used atmosphere. For more prominent directional sources that also emit a lot of low frequency sound like helicopters, the curved path results are likely to have more influence.

\section{Conclusion}

A new framework that allows the synthesis of aircraft flyover noise through a non-homogeneous atmosphere is in place at NLR and NASA. Although it is known that the curved paths will affect aircraft sound, inclusion of curved path atmospheric propagation in a synthesis environment is not trivial. The new framework emerged after consideration of several options. Ultimately it was settled on not only for its ability to perform the simulation now, but also because it is amenable to real-time implementation in the future. Through the use of new and existing codes, the new framework allows simulation of aircraft flyovers in non-homogeneous atmospheres.

It is shown that the propagation losses, emission and receiver angles are affected by the curved path. This is especially true at low elevation angles of the aircraft and relatively large distances. For the case considered, the differences in emission angle and total losses diminished at elevation angles greater than around 5 deg. and ranges less than $2000 \mathrm{~m}$. The receiver angle is more sensitive with deviations indicated up to about 10 deg. elevation angle and ranges up to $1000 \mathrm{~m}$. Based on this, it is concluded that if the aircraft elevation angle, with respect to the listener, is greater than 10 degrees, curved path effects due to refraction do not cause prominent audible differences compared to straight path results. This result is atmosphere dependent, so different atmospheric conditions might show more (or less) of an effect. Additional research is necessary to see if this conclusion may be generalized, and how it may be affected at different flight altitudes, and by cross-winds, gusting conditions, and diffraction around non-flat terrain.

For this flyover case it is clear that the most changes due to refractive effects are under relative shallow elevation angles. This is also reflected by the absence of major impact by the curved path assumption on the noise metrics. However, if the aircraft does not fly directly over a listener position the effect of atmospheric propagation is expected to make a more pronounced difference. Perhaps there are "smart ways" to use shadow zones as noise minimizing measures to reduce the noise impact in communities near airports. A future study to this end is one of the desired uses of this new capability.

Finally, a real-time implementation of the framework is envisaged in the coming years. There are several steps that can be included to speed up the current implementation. The current implementation was prototyped in Matlab, and greater efficiency can be gained by porting it to a lower level programming language, e.g. $\mathrm{C}++$. The real bottleneck however is in the calculation of the eigenrays. Two ideas have emerged from this study which can help reduce the computational burden. The first is to speed up the algorithm by starting each new trajectory point with the previous solution. Currently each new ray-tracing calculation is started without regard to the prior solution. The second idea is to take advantage of the parallelism offered by graphics processing units (GPU) over a standard CPU. Since each ray calculation is independent, the increase in speed for each ray bundle is proportional the number of processors employed.

\section{Acknowledgments}

This work was performed under a cooperative agreement between NASA and the NLR for collaborative research in community response to aircraft flyover noise, with support from the Subsonic Rotary Wing project of the NASA Fundamental Aeronautics Program. The authors thank the National Institute of Aerospace (NIA) in Hampton, Virginia for hosting an exchange visit by Michael Arntzen, and also to Dr. William Chapin of AuSIM, Inc. for 
helpful discussions. The authors also thank Mr. Aric Aumann of Analytical Services \& Materials, Inc. for his participation in thought experiments during framework architecture development and for preparing tonal samples.

\section{References}

1"National Aeronautics Research and Development Plan," National Science and Technology Council, Washington, DC, February 2010.

2"European Aeronautics: A Vision for 2020," Advisory Council for Aeronautics Research in Europe, January 2001.

${ }^{3}$ Lopes, L.V. and Burley, C.L., "Design of the next generation aircraft noise prediction program: ANOPP2," 17th AIAA/CEAS Aeroacoustics Conference, AIAA 2011-2854, Portland, Oregon, 2011.

${ }^{4}$ Boeker, E.R., "Integrated Noise Model (INM) Version 7.0 Technical Manual," Federal Aviation Administration, Washington, D.C., 2008.

${ }^{5}$ Bertsch, L., Dobrzynski, W., and Guérin, S., "Tool development for low-noise aircraft design," AIAA Journal of Aircraft, Vol. 27, No. 2, 2010, pp. 694-699.

${ }^{6}$ Bertsch, L., Guérin, S., Looye, G., and Pott-Pollenske, M., "The parametric aircraft noise analysis module - status overview and recent applications," 17th AIAA/CEAS Aeroacoustics Conference, AIAA-2011-2855, Portland, OR, 2011.

${ }^{7}$ Erkelens, L.J.J., "Research into new noise abatement procedures for the 21 st century," AIAA Guidance, Navigation, and Control Conference, AIAA-2000-4474, Denver, CO, 2000, pp. 329.

${ }^{8}$ Rizzi, S.A., Sullivan, B.M., and Sandridge, C.A., "A three-dimensional virtual simulator for aircraft flyover presentation," ICAD 2003, Proceedings of the 9th International Conference on Auditory Display, Boston, MA, 2003, pp. 87-90.

${ }^{9}$ Rizzi, S.A. and Sullivan, B.M., "Synthesis of virtual environments for aircraft community noise impact studies," 11th AIAA/CEAS Aeroacoustics Conference, AIAA-2005-2983, Monterey, CA, 2005.

${ }^{10}$ Rizzi, S.A., Sullivan, B.M., and Aumann, A.R., "Recent developments in aircraft flyover noise simulation at NASA Langley Research Center," NATO Research and Technology Agency AVT-158 "Environmental Noise Issues Associated with Gas Turbine Powered Military Vehicles" Specialists' Meeting, Paper 17, Montreal, Canada, 2008, NATO RTA Applied Vehicle Technology Panelpp. 14.

${ }^{11}$ Rizzi, S.A., Aumann, A.R., Allen, M.P., Burdisso, R., and Faller II, K.J., "Simulation of rotary and fixed wing flyover noise for subjective assessments (Invited)," 161st Meeting of the Acoustical Society of America, Seattle, WA, 2011.

${ }^{12}$ Allen, M.P., Rizzi, S.A., Burdisso, R., and Okcu, S., "Analysis and synthesis of tonal aircraft noise sources," $18 \mathrm{th}$ AIAA/CEAS Aeroacoustics Conference, Colorado Springs, CO, 2012.

${ }^{13}$ Arntzen, M., Visser, H.G., Simons, D.G., and Veen, T.A., "Aircraft noise simulation for a virtual reality environment," $17 t h$ AIAA/CEAS Aeroacoustics Conference, AIAA 2011-2853, Portland, OR, 2011.

${ }^{14}$ Janssens, K., Vecchio, A., and Van der Auweraer, H., "Synthesis and sound quality evaluation of exterior and interior aircraft noise," Aerospace Science and Technology, Vol. 12, No. 1, 2008, pp. 114-124.

${ }_{15}$ "Aircraft noise prediction program (ANOPP)," ANOPP-L30v2, NASA Langley Research Center, April, 2012.

${ }^{16}$ Begault, D.R., 3-D sound for virtual reality and multimedia. Academic Press, Inc., Chestnut Hill, MA, 1994.

${ }^{17}$ Pulkki, V., "Virtual Sound Source Positioning Using Vector-Base Amplitude Panning," Journal of the Audio Engineering Society, Vol. 45, No. 6, 1997, pp. 456-466.

18"GoldServe, AuSIM3D Gold Series Audio Localizing Server System, User's Guide and Reference, Rev. 1d," AuSIM Inc. Mountain View, CA, October 2001.

19"Prediction of sound attenuation in a refracting turbulent atmosphere with a Fast Field Program," ESDU 04011 , May 2004.

${ }^{20}$ Glassner, A.S., ed. An introduction to ray tracing. Academic Press, New York, 1993.

${ }^{21}$ Salomons, E.M., Computational atmospheric acoustics. Kluwer Academic Publishers, 2001.

${ }^{22}$ Stull, R.B., An introduction to boundary layer meteorology. Kluwer Academic Publishers, Dordrecht, Netherlands, 1987.

${ }^{23}$ Zölzer, U., ed. DAFX - Digital audio effects. John Wiley \& Sons, Ltd., West Sussex, England, 2002.

${ }^{24}$ Delany, M.E. and Bazley, E.N., "Acoustical properties of fibrous absorbent materials," Applied Acoustics, Vol. 3, No. 2, April 1970, pp. 105-116.

${ }^{25}$ Weir, D., ed., "Engine validation of noise and emission reduction technology, Phase I," NASA CR-2008-215225, 2008.

${ }^{26}$ Hubbard, H.H., ed. Aeroacoustics of flight vehicles: theory and practice. Vol. 2: Noise control. NASA-RP-1258, WRDC-TR90-3052, August 1991.

27"Atmospheric soundings," http://weather.uwyo.edu/upperair/sounding.html, University of Wyoming, College of Engineering, Department of Atmospheric Science, 2012.

${ }^{28}$ "Standard values of atmospheric absorption as a function of temperature and humidity," Society of Automotive Engineers, Inc., Aerospace Recommended Practice No. 866A, Warrendale, PA, March, 1975.

${ }^{29}$ Stone, J.R., Krejsa, E.A., and Clark, B.J., "Jet noise moddeling for coannular nozzles including the effect of chevrons," NASA CR-2003-212522, 2003.

${ }^{30}$ Heidmann, M.F., "Interim prediction method for fan and compressor source noise," NASA TM X-71763, 1979.

${ }^{31}$ Hough, J.W. and Weir, D.S., "Aircraft noise prediction program (ANOPP) fan noise prediction for small engines," NASA Contractor Report 198300, April, 1996. 